
cultures

Les cahiers de l'Acedle

14-1 | 2017

Notions en questions - Emergentisme

\title{
Langues et langage dans un paradigme enactif
}

Joëlle Aden

\section{OpenEdition}

\section{Journals}

Édition électronique

URL : http://journals.openedition.org/rdlc/1085

DOI : $10.4000 /$ rdlc. 1085

ISSN : 1958-5772

Éditeur

ACEDLE

\section{Référence électronique}

Joëlle Aden, «Langues et langage dans un paradigme enactif », Recherches en didactique des langues et des cultures [En ligne], 14-1 | 2017, mis en ligne le 19 décembre 2016, consulté le 30 avril 2019. URL : http://journals.openedition.org/rdlc/1085; DOI : 10.4000/rdlc.1085

Ce document a été généré automatiquement le 30 avril 2019

\section{(c) (i) $\odot$}

Recherches en didactique des langues et des cultures is licensed under a Creative Commons AttributionNonCommercial-NoDerivatives 4.0 International License 


\section{Langues et langage dans un paradigme enactif}

Joëlle Aden

\section{Introduction}

1 La notion d'émergentisme, soumise à la réflexion des chercheurs en didactique des langues lors de cette journée d'étude, s'inscrit dans un tournant épistémologique significatif pour les sciences cognitives contemporaines. Elle s'appuie sur l'observation des propriétés physiques, chimiques ou biologiques du vivant ${ }^{1}$ qui montrent que tout système complexe auto-produit des propriétés nouvelles. Celles-ci transforment le système et le font évoluer, de façon imprévisible, à différents niveaux de son organisation.

2 Le courant émergentiste s'est développé au cœur de «la triple révolution quantique, biologique et informatique (qui) a changé notre regard sur ce que nous appelons le 'réel' » (Trocmé-Fabre, 2013). En démontrant la complexité du vivant, la révolution quantique révèle les failles du courant positiviste et de la pensée binaire; la révolution neurobiologique, quant à elle, rend caduque le débat entre l'inné et l'acquis et, tout en montrant la plasticité du cerveau, elle met en lumière le tissage phylogénétique et ontogénétique du biologique, de l'affectif et du cognitif. Par conséquent, une matrice transdisciplinaire est devenue indispensable pour explorer et mettre en lien les caractéristiques cognitives du vivant et pour répondre au défi de la complexité, notamment dans le champ de l'enseignement-apprentissage (Nicolescu, 1996).

Dans ce texte, je présente quelques grands traits du paradigme de l'enaction (Varela, 1996) que j'ai choisi comme cadre structurant pour l'apprentissage des langues. Je mentionnerai la place particulière que tiennent les langues au cœur du langage et j'évoquerai les principales implications didactiques en lien avec ce paradigme. 


\section{Enaction et émergentisme : contexte épistémologique}

Dès $1989^{2}$, Varela lui-même présente son paradigme comme une extension de l'émergentisme. Mais si nous nous plaçons dans un cadre plus large, nous voyons que la perspective enactive s'est développée à partir d'une constellation de théories des systèmes complexes. La complexité est un principe organisationnel du vivant régi par les différents niveaux d'interaction entre les composants des systèmes que les méthodes scientifiques positivistes, focalisées sur des liens de causalité, ne peuvent expliquer de façon globale. Trois domaines scientifiques ont apporté une contribution majeure aux théories de la complexité: la théorie des systèmes qui est une formalisation mathématique de la complexité, la cybernétique qui étudie le contrôle des systèmes et l'intelligence artificielle qui cherche à les répliquer.

5 Alors que les théories scientifiques modernes postulent un équilibre linéaire et causal entre les phénomènes, les recherches tant en mathématiques qu'en physique ou en chimie mettent en évidence le comportement contre-intuitif des systèmes non linéaires qui s'organisent en boucles rétroactives imprévisibles comme dans l'exemple de la théorie du chaos ${ }^{3}$. La théorie des réseaux, plus récente, apporte une contribution cruciale, en s'appuyant sur le langage mathématique combiné à d'immenses collectes de corpus qui aident, grâce aux outils informatiques, à formaliser de façon plus concrète la complexité et l'imprévisibilité de l'évolution des systèmes ${ }^{4}$. Enfin, la branche qui étudie les systèmes adaptatifs et l'auto-organisation intéresse particulièrement la cognition humaine. Dans son Invitation aux sciences cognitives, Varela (1996) dresse un panorama des courants qui cherchent à expliquer la cognition humaine. Il montre comment les neurosciences, l'intelligence artificielle, la linguistique, la psychologie cognitive et la philosophie s'organisent en trois courants successifs ${ }^{5}$ qui « ne doivent pas être compris comme des opposés logiques (ou dialectiques) : ils représentent plus le particulier et le général, le local et la catégorie plus large » (id.: 122). Ainsi, l'enaction, le plus englobant de ces courants, prend en compte «le contexte immédiat et les effets de l'historique biologique et culturel sur la cognition et sur l'action » (id. : 119). 


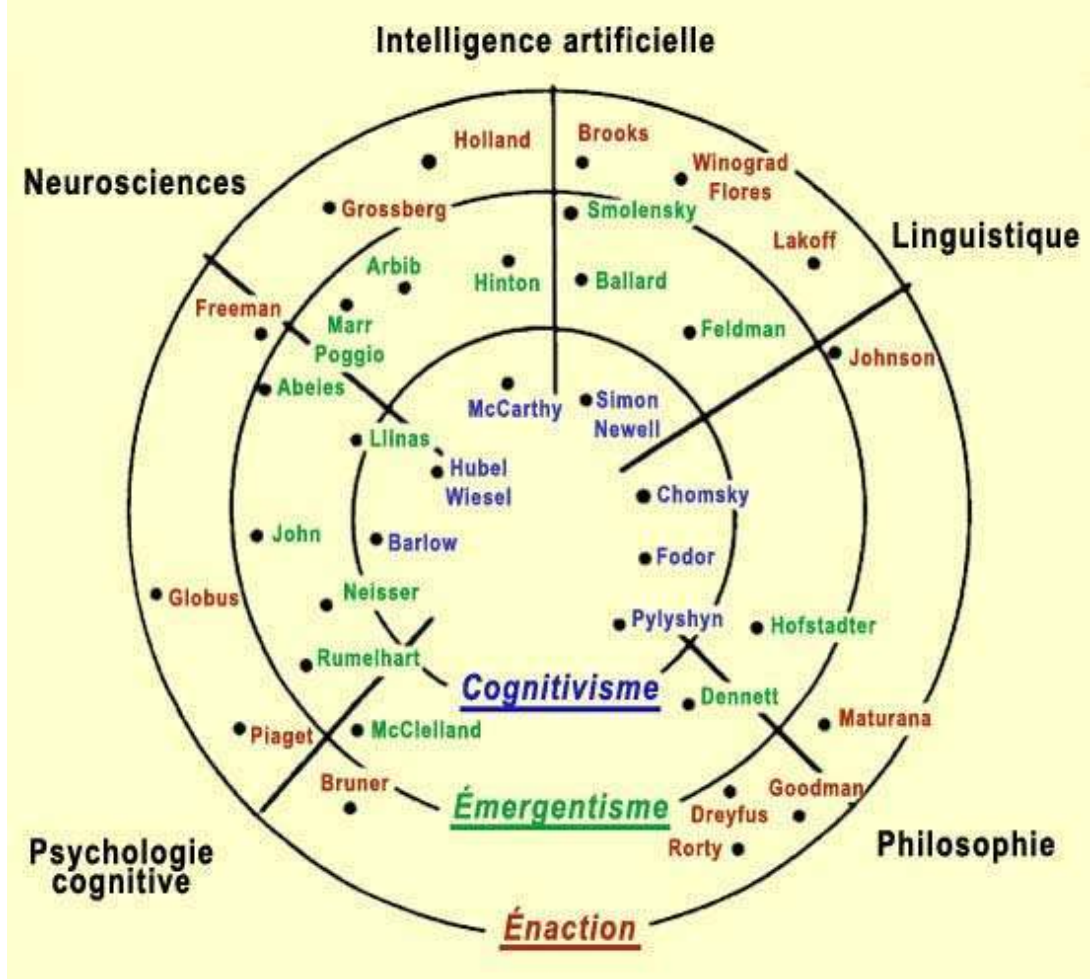

\section{En quoi l'enaction se différencie-t-elle des courants cognitivistes et connexionnistes?}

Nous proposons le terme d'enaction [de l'anglais to enact: susciter, faire advenir, faire émerger], dans le but de souligner la conviction croissante selon laquelle la cognition, loin d'être la représentation d'un monde pré-donné, est l'avènement conjoint d'un monde et d'un esprit à partir de l'histoire des diverses actions qu'accomplit un être dans le monde. (Varela et al., 1993 : 35)

Cette citation apporte un double éclairage à notre question: Varela fait d'abord l'hypothèse que c'est le double flux d'action/perception du sujet et de son environnement qui créé le monde, c'est ce qu'il appelle le couplage structurel. La seconde hypothèse découle de la première : si la connaissance advient dans l'interaction sensorimotrice du sujet avec l'environnement, alors elle n'est pas pré-donnée, mais elle émerge dans l'interaction. Ainsi, apprendre ne consiste pas à découvrir intellectuellement un monde prédéfini, mais à faire émerger un «monde (qui) se manifeste à travers l'enaction des régularités sensorimotrices » (Varela, 1996: VI). Le rejet de la notion de représentation est donc central pour comprendre le paradigme de l'enaction. Au cœur de sa philosophie, Varela remet en cause un postulat, qu'il considère comme un « préjugé » de la tradition scientifique moderne, selon lequel le monde que nous percevons serait indépendant du sujet qui le perçoit. Pour lui, au contraire, le monde et le sujet percevant se codéterminent :

Si nous devons au contraire conclure que la cognition ne peut être adéquatement comprise sans le sens commun, qui n'est autre que notre histoire physique et sociale, il nous faut en déduire que celui qui sait et ce qui est su, le sujet et l'objet, 
sont la spécification réciproque et simultanée l'un de l'autre. En termes

philosophiques : le savoir est ontologique. (Varela, 1996 : 99)

Dans les approches cognitiviste et connexionniste, «le critère d'évaluation de la cognition reste la représentation adéquate d'un monde pré-déterminé » (id. : 90-91). C'est pour illustrer l'idée que le concept d'action domine sur celui de représentation, que Varela créé ce néologisme, avec prudence néanmoins, puisqu'il note en 1989 « l'appellation (enaction) est loin d'être établie. Je la suggère ici pour les besoins de la discussion. Jusqu'à ce qu'on en propose une autre qui soit plus adéquate» (id. : 93). Il la conservera jusqu’à sa disparition.

\section{Le sens fort et le sens faible de la notion de représentation}

8 En didactique des langues, nous nous appuyons sur la notion de représentation car il est admis que les langues naturelles reflètent les représentations socialement construites du monde que nous partageons. Il s'agit là de ce que Varela appelle «le sens faible » et non controversé du concept de représentation :

Ce sens est purement sémantique : il réfère à tout ce qui peut être compris comme étant à propos de quelque chose. C'est là la définition en tant qu'interprétation puisque rien n'est à propos de quelque chose sans être d'abord interprété comme étant dans tel ou tel état (...) les mots sur une page représentent des phrases qui, à leur tour représentent - ou sont à propos de - autre chose encore. (...) Si par exemple, nous nous intéressons aux langages dans un cadre plus formel, nous pouvons dire que les énoncés d'un langage représentent leurs conditions de satisfaction. (id.:100)

Nous voyons que la représentation est «faible » quand elle est pragmatique et qu'elle correspond à une interprétation partagée qui ne véhicule aucune implication épistémologique ou ontologique; on peut partager des mots représentant des valeurs sans se préoccuper de la façon dont ces valeurs acquièrent leur signification.

La représentation au sens "fort» du terme, irrecevable pour Varela, est celle des courants représentationnistes qui postulent que « le monde est prédéfini, c'est à dire que ses propriétés sont établies préalablement à toute activité cognitive » (id. : 101).

11 C'est sans doute la clé du paradigme la plus radicalement contre-intuitive pour la communauté des éducateurs et des chercheurs en pédagogie et en didactique. En effet, les théories cognitives prennent généralement comme postulat de départ l'idée que le monde est prédéfini, que les humains, parachutés dans ce monde, ne peuvent survivre et évoluer :

qu'à la condition de posséder une carte (de ce monde) et d'apprendre à agir en fonction de celle-ci. En terme scientifique, cela veut dire que la carte est un système de représentation qui est soit inné (à propos de l'espace, du temps, des formes et des odeurs), soit acquis par apprentissage (les schémas moteurs et émotionnels) et mis à jour (l'acquisition d'une langue spécifique ou d'une tâche). (id. : 102)

À ses détracteurs constructivistes qui lui opposent le constat que les représentations sont bien des processus actifs et non un simple reflet du monde, Varela répond que dans leur modèle, l'activité cognitive reste néanmoins extrinsèque à l'environnement même si le sujet le découvre activement. Varela nous invite donc à un changement de perspective radical en sortant d'un questionnement binaire : qui est premier? Le monde ou l'image que je me fais du monde? Il est vain de chercher à répondre à une telle question, mais si 
l'on change de perspective, on peut voir que l'un et l'autre se définissent mutuellement. Varela propose donc un positionnement scientifique qu'il nomme "la voie moyenne": " c'est cet accent sur la co-détermination (au-delà de la poule et de l'œuf) qui démarque le point de vue de l'enaction de toute forme de constructivisme ou de néo-kantisme biologique » (id. : 105).

\section{Le primat de la perception et le rôle du corps en action dans la cognition}

13 La question des images que nous nous faisons du monde nous ramène inévitablement à la façon dont nous le percevons. C'est au moyen de nos sens que nous nous relions au monde et aux autres. Or les neurosciences ont, depuis la seconde moitié du $\mathrm{XX}^{\mathrm{e}}$ siècle, fait des avancées très importantes sur la compréhension de nos systèmes de perception. Nous savons aujourd'hui que notre cerveau est capable de créer sa propre réalité à partir des contraintes de son environnement; il est capable d'inhiber ou de faire surgir certaines perceptions du monde. Si l'on prend pour illustration la perception visuelle, il est courant de penser que les stimuli pénètrent par les yeux et sont transmis au cortex visuel qui les interprète, mais cette explication ignore le rôle déterminant d'une région de notre cerveau, le CGL (corps genouillé latéral) : $80 \%$ de tout ce qu'une cellule du CGL voit passer vient du dense réseau qui la relie aux autres régions du cerveau et $20 \%$ seulement vient de la rétine. Ainsi, la perception visuelle intervient dans l'élaboration de l'image qui se forme dans notre cortex visuel mais de façon modeste par rapport aux connexions qui viennent des autres parties du cerveau, et il en va de même pour tous nos sens. Ce que nous percevons est en lien avec notre environnement mais dépend de la coopération entre plusieurs réseaux du cerveau. Ces connexions permettent l'interprétation de l'objet à la fois en fonction de l'image rétinienne et en fonction de l'expectative de ce que l'objet est censé être (id.: 105-108). Ainsi, ce que nous percevons est indissociable de notre contexte, de notre histoire sociale et physique et tout enseignant devrait avoir présent à l'esprit que les supports qu'il propose aux apprenants font l'objet d'autant d'interprétations phénoménologiques qu'il a d'apprenants dans son groupe. Il n'y a pas de correspondance directe entre le mot qui désigne, par exemple « une table » et le couplage structurel auquel renvoie ce mot pour le sujet qui l'utilise.

En fait, il existe un nombre illimité de manières par lesquelles les interactions récurrentes visant à coordonner des comportements peuvent être établies entre des organismes (table, mesa, Tafel). Ce qui est pertinent est la coordination d'actions qu'elles provoquent et non la forme qu'elles adoptent. En effet, les domaines linguistiques émergent comme une dérive culturelle dans un système social, sans plan préétabli. (Maturana \& Varela, 1994 : 203)

Le passage par le jeu théâtral, que nous préconisons (voir la dernière partie de cet article), permet notamment d'associer les langues apprises à des coordinations d'actions. En effet, la perception d'un nouveau sens n'est pas simplement mentale, elle est rendue possible par la collaboration du corps et du cerveau en action: le local et le global sont inséparables (Varela, 2002: 166). Il faut, pour Varela, briser l'image d'une fonction cognitive basée dans le cerveau. Le cerveau est un système intégré à notre biologie et non un ordinateur et il n'est pas possible de comprendre la cognition si l'on s'abstrait de son incarnation (id.). Ainsi, toute forme de mentalisation est encorporée (embodied) dans la mesure où le cerveau est une partie du corps dont l'activité est intrinsèquement liée à 
l'action du corps propre ${ }^{6}$ dans un environnement donné : «il nous est impossible de séparer l'histoire de nos actions - biologiques et sociales - de la façon dont le monde nous apparaît » (Maturana \& Varela, 1994 : 9).

Les études sur le développement du langage chez l'enfant, depuis la gestation, montrent comment le langage et les langues s'enactent chez les humains : ils émergent dans l'enracinement mutuel et réciproque de la perception et de l'action, au travers des accordages tactiles, sonores, rythmiques de l'enfant avec son environnement (Aden, 2013). Varela rappelle que «l'intelligence la plus profonde et la plus fondamentale est celle du bébé qui acquiert le langage à partir d'un flot quotidien de bribes dispersées " (Varela, 1996: 56).

Tout ce à quoi on donne le nom d'objet - une table, des chaises, des gens, des visages, etc. - est entièrement dépendant de cette constante prise sensorimotrice. On ne peut se contenter de voir les objets posés là, comme indépendamment de nous. L'objet apparaît du fait de notre activité. Donc, en fait, les objets et nousmêmes co-émergeons, co-apparaissons (Varela, 1999 : 8).

En nommant les choses du monde pour partager une expérience avec autrui, nous nous percevons en train de percevoir en même temps que nous créons un espace social partagé. Grâce aux langues, nous inventons une mémoire commune de nos expériences du monde, nous pouvons revivre le passé, imaginer un futur, nous devenons capables de nous raconter, de laisser des traces lisibles et de développer une méta-conscience de notre être au monde. Mais la conscience n'est pas quelque chose qui se passe à l'intérieur de nous-mêmes. Pour le philosophe Alva Noe (2004) proche du paradigme de Varela, la conscience s'enacte, elle n'est pas localisable dans le cerveau, pas plus qu'on ne peut dire que la danse serait dans les muscles du danseur ou la valeur d'un billet de banque dans l'encre qui a servi à l'imprimer (id.). Les langues et toutes les formes de langage permettent cette circulation des significations dans et entre les systèmes humains.

gues, qui se sont développées à partir de la capacité à «langager », ont permis aux humains de développer un système neuronal capable de simuler et de s'observer en train d'agir et en faisant cela, de changer la configuration de l'espace et du temps puisque la langue permet de se projeter là où on n'est pas, de développer les formes de mémoires, notamment corporelle et épisodique. Les études en neurosciences ont également confirmé la capacité des humains de comprendre les états mentaux d'autrui (désirs, intentions, croyances) au travers de leur présence corporelle (mécanismes d'empathie).

\section{La communication n'est pas une transmission d'information, nous sommes DANS le langage}

Dans son article critique intitulé Le paradigme de l'enaction aujourd'hui, Olivier Penelaud (2010 : 18) estime que «Varela ne nous livre, en fait, qu'assez peu d'informations sur le fonctionnement et les processus sous-jacents de ce que devrait être la place du langage au sein de l'enaction ». Si cette remarque est juste en ce qui concerne le fonctionnement du langage, elle ne l'est pas sur la place et le rôle du langage. Dans leur ouvrage l'Arbre de la connaissance, Maturana et Varela (1994) proposent une autre hypothèse contreintuitive sur le langage: "il n'y a pas de transmission d'information dans la communication " (id.: 189). Pour interpréter ce point de vue, il nous faut revenir à leur perspective de biologistes. Selon eux, le langage a émergé à partir de ce qu'ils appellent «les couplages de troisième ordre» (id.: 175). Il faut comprendre par-là que les 
organismes autopoiétiques ont dérivé vers des organismes beaucoup plus complexes dotés d'un système nerveux (dont la structure repose fondamentalement sur les mêmes mécanismes). Ces organismes, parce qu'ils dépendent d'une reproduction sexuée, prennent nécessairement part à des interactions récurrentes. Le langage aurait donc émergé comme une condition nécessaire pour la continuité de ces organismes complexes dont la survie et celle de leur descendance dépend de leur capacité à coordonner leurs actions individuelles et en groupe. Il s'agit là de l'émergence des couplages sociaux qui produisent des comportements communicatifs. Mais Maturana et Varela réfutent une définition de l'information qui équivaudrait au transfert d'une donnée entre un émetteur et un récepteur.

Cette métaphore est fondamentalement fausse. Elle présuppose une unité qui n'est pas déterminée structurellement, dont les interactions sont instructives, comme si ce qui arrivait à un système en interaction était déterminé par l'agent perturbateur et non par la dynamique structurelle du système. Cependant il est évident, même dans la vie quotidienne, que ce n'est pas le cas dans la communication: chaque personne dit ce qu'elle dit, ou entend ce qu'elle entend en fonction de sa propre détermination structurelle; parler ne veut pas dire qu'on sera entendu. Du point de vue d'un observateur, il y a ambigüité dans toute interaction communicative. Le phénomène de communication ne dépend pas de ce qui est émis, mais de ce qui arrive à la personne qui reçoit. Et c'est bien autre chose qu'une transmission d'information. (id. : 189)

Pour Maturana et Varela, les comportements communicatifs sont innés ou acquis, c'est à dire qu'ils peuvent dépendre de l'organisme lui-même et/ou de son interaction avec le milieu dans lequel il se développe. Ils appellent «domaine linguistique» les comportements communicatifs appris qui constituent la base du langage. Si les espèces animales partagent des domaines linguistiques, Varela et Maturana estiment que les êtres humains donnent naissance à un nouveau domaine phénoménal, celui du langage. "Quand le langage émerge, les objets émergent aussi comme des distinctions linguistiques ». Plutôt que de penser que nous utilisons le langage, ces auteurs préfèrent dire que « nous sommes dans le langage, ou mieux, que nous « langageons » lorsque, par l'intermédiaire d'une action réflexive, nous faisons une distinction linguistique d'une distinction linguistique. » (id. : 204-205). Les traits du langage associés au comportement humain ont rendu possible la réflexivité (être capable de se décrire dans une situation) et la conscience de soi (être capable de se percevoir en train de percevoir). Le langage a affranchi les humains des limites temporelles et spatiales en leur permettant de décrire un ailleurs géographique ou temporel, de l'imaginer, de le créer par le partage du langage. Pour eux, « langager» signifie que le sens, que nous faisons émerger, constitue une partie de nous-mêmes et construit en partie l'environnement dans lequel nous agissons. Par le langage, nous nous co-déterminons avec les autres. Considérer l'acte de langager nous invite à nous situer bien en amont de l'analyse des langues, non pas qu'il ne faille pas les analyser pour les comprendre ou les apprendre, mais il nous faut retourner inlassablement à ce qu'Hélène Trocmé-Fabre appelle le Langage du Vivant (2013) et comprendre comme le rappelait Bourdieu (1982) que « mettre un mot pour un autre, c'est changer la vision du monde social, et par là, contribuer à le transformer ». Cette transformation de l'environnement modifie en retour la réalité pour les hommes. Cela confirme le poids du social dans la dérive de notre espèce et nous appelle à ne pas nous satisfaire du monde tel que nous le faisons émerger sans le questionner.

À force de dire que 'nous avons une maladie', que 'nous avons de la mémoire', que 'nous avons un corps', que 'nous avons soif... nos états physiques et cognitifs 
s'établissent à l'extérieur de nous et nous n'en sommes pas responsables. Et quand les choses ne vont pas comme nous le voulons, une solution si elle existe, doit provenir de l'extérieur! (Trocmé-Fabre, $2013: 20$ ) par l'action de langager que nous faisons émerger un monde commun. Nous sommes là dans une conception inédite du langage pour la pédagogie des langues.

Nous forgeons nos vies dans un couplage linguistique mutuel, non pas parce que le langage nous permet de nous révéler nous-mêmes mais parce que nous sommes constitués de langage dans un devenir continu que nous faisons émerger avec d'autres. Nous nous trouvons dans ce couplage ontogénétique, ni comme une référence pré-existante ni en référence à une origine, mais comme une transformation continue dans le devenir de notre monde linguistique, celui que nous construisons avec d'autres êtres humains. (Maturana, Varela, 1994 : 230)

\section{Implications dans le champ de l'éducation aux langues et par les langues}

Nous venons de le voir, l'enaction n'est pas une théorie du langage, c'est un paradigme de la connaissance dans lequel le langage tient une place centrale car il constitue la sève qui nous relie aux autres, à la connaissance du monde et à la connaissance de soi. C'est par le langage que nous co-créons en permanence ce monde que nous habitons. Le langage ne se réduit pas aux langues, mais à l'intérieur du langage, les langues orales et écrites reflètent le niveau de précision et d'abstraction totalement unique dont notre espèce est capable ainsi que le tissage de l'affectivité avec la connaissance.

Mettre en œuvre une pédagogie enactive dans le système éducatif n'est pas chose aisée, car cela requiert une approche systémique et globale de la connaissance qui donne un statut central aux langues. Ce paradigme nécessite a minima de décloisonner en partie les savoirs disciplinaires, de réorganiser le temps et l'espace scolaire, de sortir de la linéarité des programmes et de l'uniformisation des groupes d'âge ou de niveau. Cela suppose également de donner une place au corps et à l'affect dans les activités langagières - et pas uniquement à l'école maternelle. Il nous faudrait apprendre à associer les langages artistiques et les langages scientifiques comme des chemins complémentaires dans la coconstruction du monde, ne pas restreindre les langues à celles de l'école mais apprendre à "translangager" (Aden, 2012; 2014-b), c'est à dire à utiliser de la façon la plus performante possible les répertoires langagiers des apprenants et les questionner pour comprendre que les langues, les théories, les idéologies, sont des perspectives sur le monde. Cela signifie qu'il faut entrainer les apprenants à changer de points de vue et de "point de sentir» (Berthoz, 2004) en convoquant le développement des neurosciences cognitives et sociales, et en s'appuyant notamment sur les mécanismes d'empathie (Aden, 2008 ; 2014-a ; Aden \& Eschenauer, 2014 ; 2015). Le travail sur les langues et les langages est aussi un moyen de questionner les normes et leurs rôles dans la stabilité sociale. Il faut, pour tout cela, apprendre à sortir des enfermements ego et ethno-centrés, naviguer entre les langues, les codes et les référents, penser une éducation qui reconnaît la diversité et le potentiel de chaque enfant et qui développe la créativité et l'autonomie plutôt que la conformité et la dépendance.

Pour un tel projet pédagogique, nous ne partons pas de rien car depuis l'éducation nouvelle du début du $20^{\mathrm{e}}$ siècle, de nombreuses expérimentations dans plusieurs pays nous ont permis de collecter des observations et des analyses suffisamment pertinentes 
pour initier une nouvelle étape post-humaniste que le paradigme de l'enaction porte à l'échelle de la compréhension de notre espèce. On peut noter l'ouvrage de Masciotra \& al., (2008) qui propose des principes pédagogiques généraux pour des enseignants enactifs. Nous disposons également du référentiel cognitif d'Hélène Trocmé-Fabre (2003), L'arbre $d u$ savoir-apprendre, fondé sur une proposition transdisciplinaire des sciences du Vivant. J'ai, par ailleurs, proposé la mise en place de projets largement inspirés du référentiel de Trocmé-Fabre, pilotés par des communautés d'expériences et de pratiques auxquelles s'associent des artistes et des chercheurs. Ces communautés enactent des questionnements collaboratifs sur les langues dans les apprentissages. (Aden, 2013 ; 2014b ; Aden \& Eschenauer, 2014 ; 2016 ; Eschenauer, 2014).

Nous terminerons en indiquant quelques questions et axes de réflexions plus spécifiquement liés à la pédagogie des langues à partir des phénomènes que nous venons de décrire et que je continue à explorer avec les chercheurs de notre équipe :

- Concevoir et évaluer des situations dans lesquelles les apprenant sont DANS le langage et enactent les langues et qui permettent de tisser la maîtrise des langues avec la maîtrise émotionnelle des situations ;

- Imaginer une exploration polysensorielle et phénoménologique des langues orales (Aden \& Aden, en préparation);

- S'appuyer sur les répertoires langagiers déjà-là et intégrer des expériences sensorimotrices dans l'apprentissage des langues de la communauté en collaborant avec des artistes, acteurs, conteurs, plasticiens, danseurs ;

- Développer des compétences émotionnelles et des attitudes d'empathie kinesthésique et émotionnelle par les langages artistiques dans les langues apprises et dans toutes les langues des apprenants ;

- Stimuler la capacité à translangager, c'est-à-dire la flexibilité mentale et émotionnelle dans des situations inconnues et potentiellement déstabilisantes.

Nous cherchons particulièrement à faire émerger des projets qui reflètent des questionnements individuels et collectifs authentiques des apprenants et des enseignants, au travers de narrations qu'ils inventent, de situations de vie, d'histoires tirées de répertoires artistiques, d'extraits d'œuvres littéraires ou scientifiques dans différentes langues, époques et cultures. Ces projets sont des opportunités d'enacter la compréhension d'un monde qui s'écrit entre stabilité relative et émergence réfléchie. Intégrer des choix authentiques relève d'une éducation réflexive à la communication autonome.

\section{Conclusion : implications dans la recherche en didactique des langues}

L'émergentisme appliqué à la recherche en didactique induit la prise en compte des notions pour lesquelles il convient de trouver de nouveaux outils de collectes et d'analyse de données. La non-linéarité, par exemple, nous rappelle que l'on ne peut mesurer une évolution de classe, comparer la progression de sujets apprenant les langues, évaluer des effets extrinsèques d'un dispositif sans prendre en compte la variabilité et la temporalité de chacun des éléments de la situation envisagée. La connectivité, qui se traduit par la densité des synchronisations et des adaptations dans une situation aussi complexe que l'apprentissage d'une langue, indique que même s'il est possible de repérer des invariants 
qui semblent indiquer des tendances, rien ne permet d'envisager que ces effets soient reproductibles à l'identique pour tous sur le moyen ou le plus long terme. Or, plus les sujets sont autonomes et plus les relations sont complexes.

Certains linguistes s'intéressent également aux langues en tant qu'elles seraient des systèmes autopoïétiques; pourtant Varela lui-même a mis en garde contre une généralisation de l'autopoïèse à tous les niveaux de la complexité :

Chaque niveau - système immunitaire, cerveau, autopoïèse cellulaire - présente un mécanisme d'émergence spécifique. (...) Évidemment, vu de loin, il y a un air de famille dans le fonctionnement du système immunitaire, du cerveau, de l'autopoïèse. Mais ce n'est pas dans les généralités que l'on trouvera les réponses à nos questions. L'une des grandes erreurs est de confondre les niveaux d'organisation. Ainsi l'autopoïèse décrit-elle le fonctionnement du niveau d'organisation cellulaire. Mais le système immunitaire, le cerveau, l'organisme humain ne sont pas autopoïétiques. Ils possèdent d'autres caractéristiques d'organisation qui permettent leur émergence. (Varela, 2002 : 175-176)

Nous voyons que si des portes se sont ouvertes, il s'agit maintenant de les franchir. Varela a recherché tout au long de sa carrière à expliquer les fondements biologiques de la subjectivité et de la conscience comme expérience. Il a élaboré une proposition méthodologique, la neurophénoménologie (Varela, 1996-a), dans laquelle il institue une relation étroite entre deux méthodes: l'observation de substrats neuronaux des actes cognitifs et une exploration phénoménologique de l'expérience humaine. Il s'agissait pour lui d'apporter une réponse au «problème difficile» de Chalmers (1995) : comment certains processus cognitifs neurologiques font-ils émerger de l'expérience consciente? Or, le langage tient une place centrale dans ce questionnement.

Pour comprendre un acte mental dont la nature est expérientielle, il n'y a pas d'autre alternative que de prendre en compte l'expérience en première personne du sujet, mais ceci requiert une méthodologie inédite, "un ré-apprentissage et une maitrise de la description phénoménologique (...) afin de travailler de façon sérieuse sur la collecte de données en première personne » (id. : 346-347 ; Varela \& Shear, 1999). En résonance avec les travaux de Pierre Vermersch et de Nathalie Depraz (2000), Varela a cherché à définir les contours d'une méthode d'exploration de l'expérience en première personne. Il a quitté la scène scientifique prématurément en laissant en jachère un projet scientifique inédit et largement inexploré dans notre domaine de recherche. Il le résumait ainsi :

La méthode scientifique exige que la recherche soit rendue publique, ouverte à vérification. Or, l'expérience subjective est, par définition, privée et n'est donc accessible qu'à l'expérience personnelle. (...) Les scientifiques devraient donc créer une nouvelle science de la conscience où la subjectivité n'est ni réduite, ni occultée, mais centrale. (Varela, $2002: 163$ )

Il laisse en héritage une philosophie de l'être comme expérience et de l'expérience comme être qui s'inscrit dans une épistémologie relationnelle de la conscience enactée du monde. Le défi actuel de la recherche dans les sciences cognitives est celui de la conscience, «ce souffle immatériel qui fait de l'homme un Vivant en perpétuel questionnement dans un univers où fiction et réalité se répondent » $\left(\mathrm{Aden}^{7}\right)$. Le chemin qui va de la recherche fondamentale à la recherche appliquée en éducation sur ces questions commence tout juste à émerger ...

Prospero:

Our revels now are ended. These our actors,

As I foretold you, were all spirits, and

Are melted into air, into thin air: 
And like the baseless fabric of this vision, The cloud-capp'd tow'rs, the gorgeous palaces, The solemn temples, the great globe itself, Yea, all which it inherit, shall dissolve, And, like this insubstantial pageant faded, Leave not a rack behind. We are such stuff As dreams are made on; and our little life Is rounded with a sleep.

The Tempest, Act 4, scene 1,148-158

\section{BIBLIOGRAPHIE}

Aden, J. \& Aden, S. (en préparation). “An enactive pedagogy for language learning and the phenomenology of perceived sound". Journal of the French Association for Research on Cognition (ARCo).

Aden, J. \& Eschenauer, S. (2016). « Se construire par l'expérience esthétique théâtrale : AiLES, analyse d'une démarche énactive au collège ». Recherche en théâtre et éducation: IDEA. Paris : CNRS Sorbonne Paris 1 : p-e-r-f-o-r-m-a-n-c-e, vol. $1, \mathrm{~N}^{\circ} 2$.

Aden, J. (2014-b). "De la langue en mouvement à la parole vivante : théâtre et didactique des langues. Le Vécu corporel dans la pratique d'une langue”. Langages, n 192, pp. 101-110.

Aden, J. (2014-a). « Résonance émotionnelle et empathie : synergie entre pratiques théâtrales et langagières ». In J. Aden \& A. Arleo (dir.), Languages in motion/Langues en mouvement. Les cahiers du CRINI, Université de Nantes.

Aden, J., Eschenauer, S. (2014) « Théâtre et empathie en classe bilangue : didactiser l'émergence? ». Les Langues Modernes, 4/2014.

Aden, J. (2013). « Apprendre les langues par corps ». In O. Fertat, Omar, Y. Abdelkader, S. Bazile, Pour un théâtre-monde. Plurilinguisme, interculturalité, transmission. Bordeaux : Presses universitaires de Bordeaux, pp. 109-123.

Aden, J. (2012). « La Médiation linguistique au fondement du sens partagé : vers un paradigme de l'énaction en didactique des langues ». ÉLA, 2012/3 n 167, pp. 267-284.

Aden, J. (2008). « Compétences interculturelles en didactique des langues. Développer l'empathie par la théâtralisation ». In J. Aden (éd.), Apprentissage des langues et pratiques artistiques. Paris : Manuscrit Université, pp. 67-101.

Berthoz, A. (2004). « Physiologie du changement de point de vue ». In A. Berthoz \& G. Jorland (éds), L'Empathie. Paris : Odile Jacob, pp. 251-275.

Bourdieu, P. (1982). Ce que parler veut dire. Paris: Fayard.

Chalmers, D. (1995). « Facing up to the problem of consciousness ». Journal of Consciousness Studies , 2 (3), pp. 200-219.

Depraz, N., Varela, F. J., \& Vermersch, P. (2000). The Gesture of Awareness: An Account of its Structural Dynamics. Advances in Consciousness Research, pp. 121-137. 
Dubuc, B. Site Le Cerveau à trois niveaux, université McGill. http://lecerveau.mcgill.ca/flash/a/ a_12/a_12_p/a_12_p_con/a_12_p_con.html\#2 (dernière consultation le 6 novembre 2016).

Eschenauer, S. (soumis). "Questioning the notion of performance: effects of an aesthetic theatrical experience on translanguage learning”, The Arts in Language Teaching. International Perspectives : Performative - Aesthetic - Transversal - Europa lernen. Perspektiven für eine Didaktik europäischer Kulturstudien, LIT Verlag (Berlin, Vienna, Zurich, London) (date de publication attendue : 2016).

Eschenauer, S. (soumis). «Créativité et énaction dans les apprentissages performatifs : vivre et encorporer ses langues », Revue Recherche et Éducation (Date prévue de publication : 2017).

Eschenauer, S. (2014). « Faire corps avec ses langues. Théâtre et didactique : vers une définition de la translangageance ». In J. Aden \& A. Arleo (dir.), Languages in motion/Langues en mouvement. Les cahiers du CRINI, Université de Nantes.

Maturana, H. \& Varela, F. (1994 version française). L'arbre de la connaissance. Racines biologiques de la comprehension humaine. Editions Addison-Wesley France, S.A.

Masciotra, D., Roth, W.-M. \& Morel, D. (2008). Énaction. Apprendre et enseigner en situation. Montréal : De Boeck.

Nicolescu, B. (1996). La Transdisciplinarité. Monaco : Editions du Rocher.

Noë, A. (2004). Action in Perception. Cambridge: MIT Press.

Penelaud, O. (2010). Le paradigme de l'énaction aujourd'hui. Apports et limites d'une théorie cognitive révolutionnaire. PLASTIR 2010/1, pp. 1-38.

Trocmé-Fabre, H. (2003). L'arbre du savoir apprendre. Paris, Éditions Être et Connaître.

Trocmé-Fabre, H. (2013) Le langage du vivant : une voix, une voie en sommeil ? Paris : Ed. Précursions.

Varela, F. (2002). « Autopoïese et émergence ». In R. Benkinrane (ed.), La complexité, vertiges et promesses. 18 histoires de science. Paris : Editions Le Pommier, pp. 161-176.

Varela, F. (1999) Quatre phares pour l'avenir des sciences cognitives, Revue Théorie Littérature Enseignement, $\mathrm{n}^{\circ}$ 17, pp. 7-21.

Varela, F \& Shear, J. (eds.) (1999). « The view from within: First Person Methods in the Study of Consciouness ", Imprint Academic, London.

Varela, F. (1996) ( $1^{\text {ère }}$ édition en 1989). Invitation aux sciences cognitives. Paris : Seuil.

Varela, F., Thompson, E., Rosch, E. (1993). L'Inscription corporelle de l'esprit : sciences cognitives et expérience humaine. Paris, Édition du Seuil.

Varela, F. (1996-a). « Neurophenomenology. A Methodological Remedy for the Hard Problem ». Journal of Conciousness Studies, 3, $n^{\circ}$ 4, pp. 330-349.

\section{NOTES}

1. Les domaines linguistique et langagier constituant des propriétés du Vivant.

2. La première édition de l'ouvrage "Invitation aux sciences cognitives" a été publié en 1989 dans la collection "Sciences ouvertes" sous le titre: Connaître. Les sciences cognitives, tendances et perspectives. Elle a été mise à jour en 1996. 
3. Edward Lorenz présente sa théorie du Chaos en 1972 devant l'Association américaine pour le progrès des Sciences avec une question restée célèbre: "Le battement d'aile d'un papillon au Brésil peut-il déclencher une tornade au Texas ?"

4. Avec de nombreuses applications en linguistique, notamment l'étude de la structure en réseau du langage. Voir par exemple R.V. Solé \& al. Language networks : their structure, function and evolution. Complexity, vol. 15, pp. 20-26, 2009.

5. Dans la figure, l'émergentisme correspond au courant connexionniste.

6. Le corps propre désigne une unité synthétique de l'expérience de soi : à la fois le corps biologique et l'expérience sensible intime de ce corps biologique en tant qu'il constitue une identité dans un environnement.

7. Non publié

\section{RÉSUMÉS}

Dans cet article, je situe le paradigme enactif (Varela) au sein du courant émergentiste et je montre ce qui le différencie des approches cognitiviste et connexionniste. L'enaction met en lumière le rôle du corps en action et de la perception dans la cognition et attribue une place primordiale à l'acte de langager tissé avec l'affect dans le développement humain. Je termine par un survol de quelques implications didactiques de ce paradigme pour l'éducation aux langues. Des applications précises sont à trouver dans des publications en référence.

In this paper, I describe the enaction paradigm within an emergentist framework and I draw attention to what differentiates it from cognitive and connexionist approaches. Enaction highlights the role of action and perception in cognition and stresses the importance of languaging and affect in human development. I conclude with an overview of implications for languages education. Specific teaching applications are to be found in the references.

\section{INDEX}

Mots-clés : langager, langage corporel, émotions, enaction

Keywords : languaging, body language, emotions, enaction

\section{AUTEUR}

\section{JOËLLE ADEN}

Université Paris-Est Créteil, France, EA 3958 IMAGER

Joëlle Aden est professeure des universités à l'Espé de l'Université Paris-Est Créteil où elle enseigne la méthodologie de la recherche en didactique des langues et des approches plurielles. Dans ses travaux sur l'éducation aux langues et par les langues, elle explore le paradigme de l'énaction de Varela en s'appuyant notamment sur l'expérience artistique et esthétique, principalement le théâtre. Elle travaille sur le rôle des émotions et de l'empathie dans les interactions de classe. Elle a proposé la notion de «translangager » dans une acception neuro- 
phénoménologique. Les doctorants qu'elle accompagne formalisent les liens langues/ langages/ expérience esthétique au travers de différentes formes de pratiques artistiques.

Courriel : joelle.aden [at] u-pec.fr

Toile : https://univ-paris12.academia.edu/JoelleAden 